Mohamed SARMOUM ${ }^{1,2}$

Frédéric GUIBAL ${ }^{3}$

\title{
Effet des facteurs stationnels sur la croissance radiale et la réponse du pin d'Alep au climat dans le massif de l'Ouarsenis, Algérie
}

Fatiha ABDOUN ${ }^{1}$

${ }^{1}$ Université des sciences et de la technologie Houari Boumediene (USTHB)

Faculté des sciences biologiques Laboratoire d'écologie végétale et environnement BP 32, El Alia, Bab Ezzouar 16111 Alger

Algérie

${ }^{2}$ Université Ibn Khaldoun

Faculté des sciences de la nature et de la vie BP P 78, Zaâroura 14000 Tiaret

Algérie

${ }^{3}$ Aix-Marseille Université (AMU)

UMR 7263 CNRS-IRD-UAPV Institut méditerranéen de biodiversité et d'écologie marine et continentale (IMBE) Jardin du Pharo

58, boulevard Charles Livon 13284 Marseille Cedex 07 France

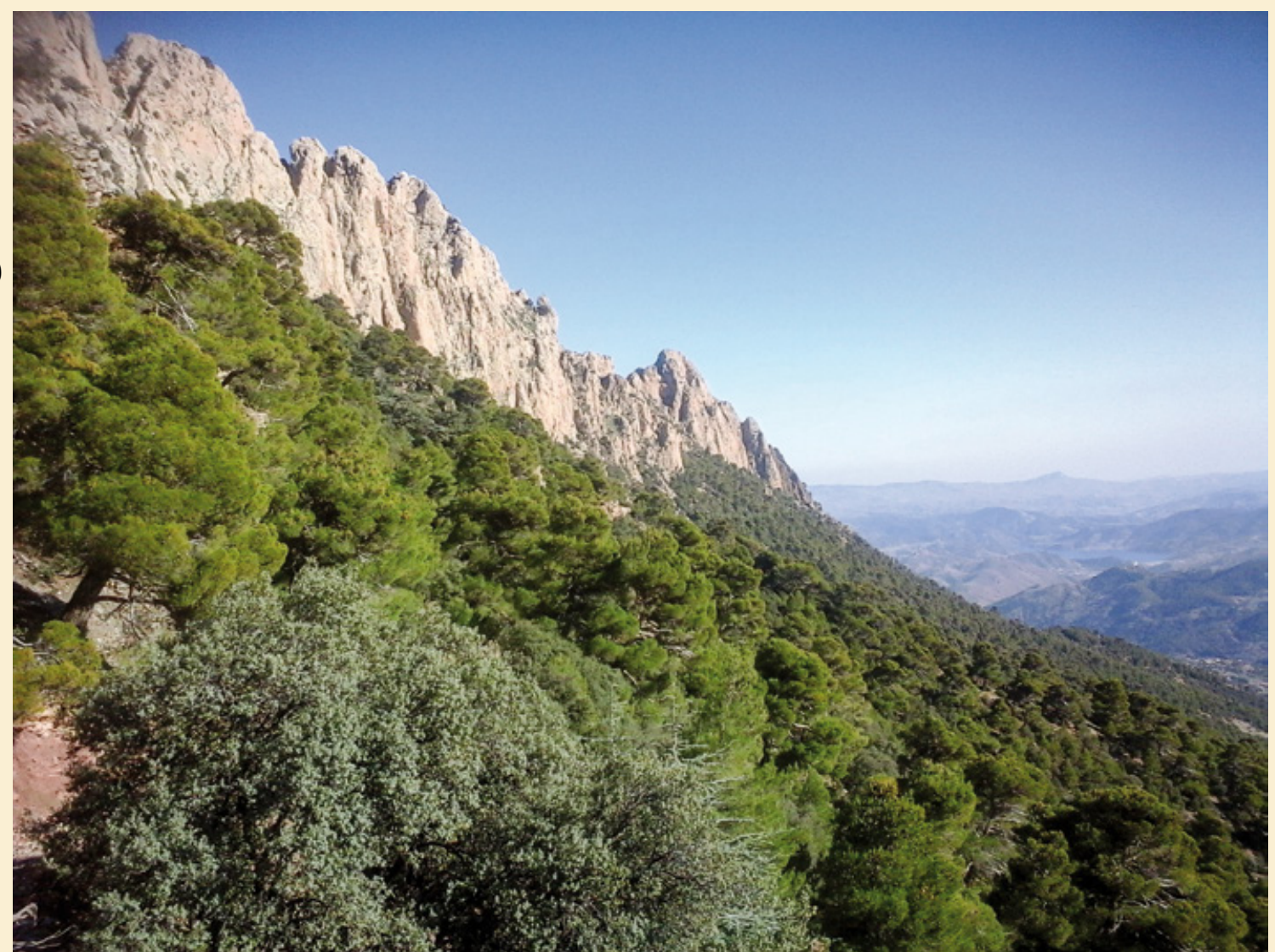

Photo 1.

Vue d'ensemble de la zone culminante de l’Ouarsenis où de vastes pinèdes se développent sur substrat calcaire. Photo M. Sarmoum. 


\section{RÉSUMÉ}

\section{EFFET DES FACTEURS STATIONNELS SUR LA CROISSANCE RADIALE ET LA RÉPONSE DU PIN D'ALEP AU CLIMAT DANS LE MASSIF DE L'OUARSENIS, ALGÉRIE}

Les relations entre le climat et la croissance radiale sur la période 1967-2010 de neuf populations de pin d'Alep dans le massif de l'Ouarsenis (Nord-Ouest de l'Algérie) ont été étudiées en fonction des facteurs stationnels (substrat, altitude, exposition et âge des arbres) à l'aide de méthodes de dendrochronologie. Les variations interannuelles des épaisseurs de cernes ont été analysées. L'étude rétrospective de la croissance radiale indique une forte variabilité interannuelle des épaisseurs des cernes à l'intérieur d'une même population. Les paramètres statistiques calculés indiquent une forte variabilité de la croissance radiale entre les populations, cette variabilité étant liée aux facteurs stationnels et à l'âge des arbres. L'âge joue un rôle négatif sur l'épaisseur moyenne des cernes et sur le phénomène de persistance. La sensitivité (sensibilité au climat) moyenne des arbres tend à être plus élevée sur les substrats gréseux ou schisteux que sur les substrats calcaires. L'analyse des relations cerne-climat montre que le pin d'Alep est réactif aux précipitations de la période précédant ou contemporaine de la formation du cerne. Les températures interviennent négativement sur la croissance radiale, surtout par leurs valeurs maximales. Les relations du pin d'Alep aux conditions climatiques sont modulées par les facteurs stationnels, notamment le substrat. Les populations les plus réactives se localisent davantage sur un substrat gréseux. Ces résultats constituent un descriptif utile pour comprendre la plasticité écologique du pin d'Alep et sa réponse aux changements climatiques, afin de proposer des mesures destinées à assurer une meilleure protection des pinèdes menacées depuis plusieurs décennies.

Mots-clés: Pinus halepensis, facteurs stationnels, croissance radiale, relations cerne-climat, sécheresse, Ouarsenis.

\section{ABSTRACT}

\section{EFFECTS OF STATION-SPECIFIC FACTORS ON RADIAL GROWTH AND RESPONSES TO CLIMATE IN ALEPPO PINES IN ALGERIA'S OUARSENIS RANGE}

The relationships between climate and radial growth from 1967 to 2010 in nine Aleppo pine stands in the Ouarsenis range (north-western Algeria) were studied according to station-specific factors (substrate, altitude, exposure and age of the trees) using dendro-chronological methods, with an analysis of interannual variations in the thickness of growth rings. A retrospective analysis of radial growth showed high interannual variability in growth ring thickness within the same stand. The statistical parameters calculated showed highly variable radial growth between the different stands, which was related to station-specific factors and to tree age. Average growth ring thickness and persistence declined with the age of the trees. Average sensitivity to climate tended to be greater in trees growing on sandstone or schist substrates than on limestone. Our analysis of the relationships between climate and growth rings shows that the Aleppo pine is responsive to precipitation before or during the formation of growth rings. Higher temperatures, especially when they reach their maximum, have a negative effect on radial growth. The relationships between Aleppo pines and climatic conditions are modulated by station-specific factors, especially the substrate. The most responsive stands tend to be growing on a sandstone substrate. These results provide a useful description to help understand the ecological flexibility of the Aleppo pine and how it responds to climate change, with a view to proposing better ways of protecting pine forests that have been under threat for several decades.

Keywords: Pinus halepensis, station-specific factors, radial growth, relationships between growth rings and climate, drought, Ouarsenis.

\section{EFECTO DE LOS FACTORES DE SITIO EN EL CRECIMIENTO RADIAL Y RESPUESTA DEL PINO CARRASCO AL CLIMA EN EL MACIZO DE OUARSENIS, ARGELIA}

Se estudiaron las relaciones entre clima y crecimiento radial de nueve poblaciones de pino carrasco en el macizo montañoso de Ouarsenis (Noroeste de Argelia), en el período 1967-2010, atendiendo a factores de sitio (sustrato, altitud, exposición y edad de los árboles) y aplicando métodos de dendrocronología. Se analizaron las variaciones interanuales del ancho de los anillos. El estudio retrospectivo del crecimiento radial indica una alta variabilidad interanual del ancho de los anillos entre individuos de una misma población. Los parámetros estadísticos calculados reflejan indican una alta variabilidad del crecimiento radial entre las poblaciones, dicha variabilidad está vinculada a los factores de sitio y a la edad de los árboles. La edad incide negativamente en el ancho promedio de los anillos y en el fenómeno de persistencia. La sensibilidad climática media de los árboles tiende a ser mayor en los sustratos de areniscas o esquistos que en los calizos. El análisis de las relaciones anillo-clima muestra que el pino carrasco es reactivo a las precipitaciones del período precedente o contemporáneo a la formación del anillo. Las temperaturas intervienen negativamente en el crecimiento radial, especialmente sus valores máximos. Las relaciones del pino carrasco con las condiciones climáticas se ven moduladas por los factores de sitio, principalmente el sustrato. Las poblaciones más reactivas se ubican preferentemente en sustratos de arenisca. Estos resultados proporcionan información útil para comprender la plasticidad ecológica del pino carrasco y su respuesta a los cambios climáticos con el fin de proponer medidas encaminadas a garantizar una mejor protección de pinares que llevan varias décadas amenazados.

Palabras clave: Pinus halepensis, factores de sitio, crecimiento radial, relaciones anillo-clima, sequía, Ouarsenis. 


\section{Introduction}

Dans le contexte des changements climatiques qui prévalent actuellement, les essences forestières peuvent réagir par des changements importants dans leur répartition géographique, leur productivité et leur phénologie (Saxe et al., 2001 ; Thuiller et al., 2008 ; Charru et al., 2010 ; Jonard et al., 2014). La région méditerranéenne figure parmi les régions les plus affectées par ces changements qui risquent d'y hypothéquer l'avenir des écosystèmes forestiers et autres espaces boisés (GIEC, 2007).

Si les travaux menés sur les forêts de la rive nord de la Méditerranée sont nombreux (Giorgi et Lionello, 2008 ; Coomes et al., 2014), les études conduites sur les impacts des changements climatiques sur les essences forestières d'Afrique du Nord demeurent encore rares (Quézel, 2000 ; El Khorchani et al., 2007).

Depuis quelques décennies, l'Algérie a enregistré une tendance à la sécheresse, une augmentation des températures et un glissement des étages bioclimatiques vers l'aridité, notamment dans la partie occidentale (Benabadji et Bouazza, 2000 ; Hirche et al., 2007 ; Meddi et Toumi, 2013). Le dépérissement observé dans les cédraies méridionales depuis le début des années 1980 a été fortement corrélé avec les changements climatiques (Sarmoum, 2008 ; Messaoudene et al., 2013 ; Rabhi et al., 2014).

Malgré la présence du pin d'Alep en Algérie sous des conditions bioclimatiques très variées (Kadik, 1983 ; Bensaid, 1995), aucune étude n'a été réalisée pour comprendre sa réponse à long terme aux changements climatiques. Les études réalisées sur cette espèce sur le pourtour méditerranéen ont montré que les relations entre la croissance et le climat présentent une forte hétérogénéité d'un site à un autre (Safar, 1994 ; Nicault, 1999 ; de Luis et al., 2013) et nécessitent au préalable de prendre en compte la variabilité des conditions du milieu dans l'étude des relations cerne-climat.

Notre travail avait pour objectif d'étudier l'effet des facteurs stationnels sur la croissance radiale et la réponse du pin d'Alep aux variations interannuelles du climat sur un territoire limité au massif de l'Ouarsenis (Nord-Ouest de l'Algérie). Ce massif présente l'avantage d'offrir sur une surface restreinte une gamme de biotopes variés selon l'altitude, l'exposition et le substrat. Ce travail visait aussi à comprendre la réponse du pin d'Alep à la tendance climatique actuelle marquée par la sécheresse, et à mettre en évidence un éventuel déficit de croissance ou une éventuelle variation spatio-temporelle de la réponse des arbres au climat.

\section{Matériel et méthodes}

\section{Description de la zone d'étude}

Le massif de l'Ouarsenis est le plus vaste massif forestier de l'Algérie occidentale. Ce territoire s'étale sur une longueur de $200 \mathrm{~km}$ et une largeur de $60 \mathrm{~km}$ et constitue une transition entre la plaine littorale au nord et les hauts plateaux au sud (figure 1 ; photo 1). Ce massif est caractérisé par l'existence de plusieurs formations géologiques (Mattauer, 1958), entraînant une grande variété de substrats et de sols (Sari, 1977). Le bioclimat varie du semi-aride à l'humide selon un gradient altitudinal qui s'étend de 400 m à 1985 m. Une tendance à la sécheresse a été constatée dans cette région depuis les années 1980, avec une baisse de $25 \%$ des cumuls de précipitations annuelles et une augmentation de $0,7^{\circ} \mathrm{C}$ des températures moyennes annuelles (Sarmoum, 2008). Ces conditions de sécheresse conjuguées à une forte pression anthropique et des incendies répétés ont conduit à la dégradation des pinèdes de l'Ouarsenis et à leur fragmentation (BNEDER, 2009) (photo 2).

\section{Échantillonnage et prélèvement du matériel végétal}

L'échantillonnage a porté sur neuf stations de pin d'Alep réparties sur l'ensemble du massif (tableau I). Ces stations ont été définies sur la base des facteurs écologiques (altitude, exposition et type de sol) et de l'âge des populations. Au sein de chaque station, 15 arbres parmi les

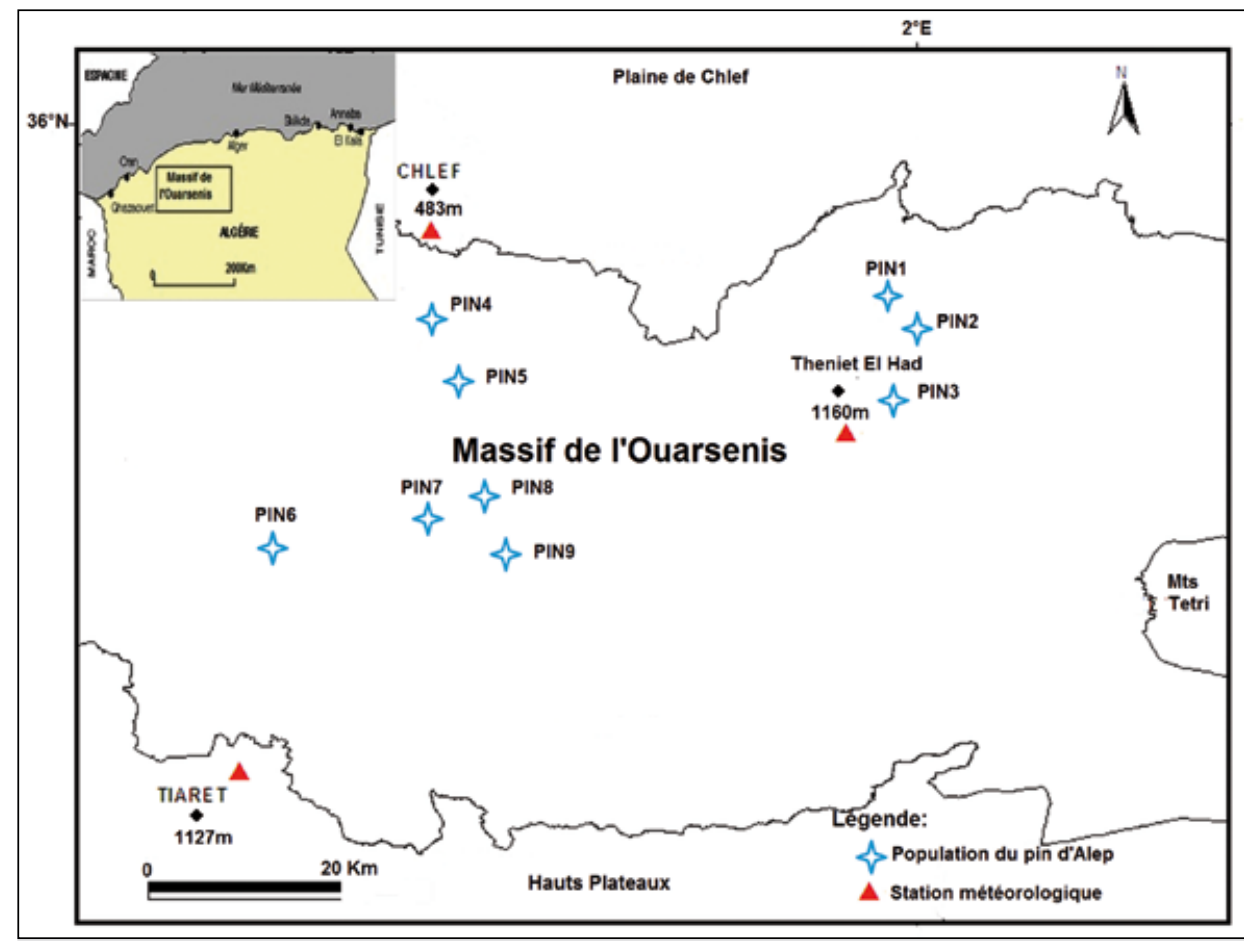

Figure 1.

Carte descriptive de la situation géographique de la zone d'étude, des populations échantillonnées et les stations météorologiques utilisées dans le cadre du présent travail. 
Tableau I.

Localisation géographique, variabilité des conditions de milieu et des peuplements échantillonnés dans la zone d'étude.

\begin{tabular}{|c|c|c|c|c|c|c|}
\hline Population & $\begin{array}{l}\text { Coordonnées } \\
\text { géographiques }\end{array}$ & $\begin{array}{l}\text { Altitude } \\
\text { (m) }\end{array}$ & Exposition & $\begin{array}{l}\text { Pente } \\
\text { (\%) }\end{array}$ & Édaphisme & $\begin{array}{c}\text { Âge } \\
\text { moyen (ans) }\end{array}$ \\
\hline PIN1 & $\begin{array}{l}35^{\circ} 52^{\prime} 54^{\prime \prime} \mathrm{N} \\
01^{\circ} 58^{\prime} 35^{\prime \prime} \mathrm{E}\end{array}$ & 1225 & NNE & $30-40$ & Colluvion de grès & 74 \\
\hline PIN2 & $\begin{array}{l}35^{\circ} 53^{\prime} 05^{\prime \prime} \mathrm{N} \\
01^{\circ} 58^{\prime} 2^{\prime \prime} 8 \mathrm{E}\end{array}$ & 1041 & NW & $40-50$ & Colluvion de grès & 51 \\
\hline PIN3 & $\begin{array}{l}35^{\circ} 52^{\prime} 50^{\prime \prime} \mathrm{N} \\
01^{\circ} 58^{\prime} 30^{\prime \prime} \mathrm{E}\end{array}$ & 1143 & NW & $30-40$ & Colluvion de grès & 60 \\
\hline PIN4 & $\begin{array}{l}35^{\circ} 53^{\prime} 28^{\prime \prime} \mathrm{N} \\
01^{\circ} 40^{\prime} 24^{\prime \prime} \mathrm{E}\end{array}$ & 1150 & NNE & $10-20$ & Calcaires & 58 \\
\hline PIN5 & $\begin{array}{l}35^{\circ} 53^{\prime} 40^{\prime \prime} \mathrm{N} \\
01^{\circ} 39^{\prime} 05^{\prime \prime} \mathrm{E}\end{array}$ & 1254 & NW & $20-30$ & Calcaires & 105 \\
\hline PIN6 & $\begin{array}{l}35^{\circ} 47^{\prime} 08^{\prime \prime} \mathrm{N} \\
01^{\circ} 29^{\prime} 51^{\prime \prime} \mathrm{E}\end{array}$ & 708 & SW & $30-40$ & Schistes & 112 \\
\hline PIN7 & $\begin{array}{l}35^{\circ} 51^{\prime} 29^{\prime \prime} \mathrm{N} \\
01^{\circ} 41^{\prime} 22^{\prime \prime} \mathrm{E}\end{array}$ & 815 & SW & $20-30$ & Calcaires & 43 \\
\hline PIN8 & $\begin{array}{l}35^{\circ} 51^{\prime} 53^{\prime \prime} \mathrm{N} \\
01^{\prime} 39^{\prime} 27^{\prime \prime} \mathrm{E}\end{array}$ & 1030 & SE & $30-40$ & Calcaires & 45 \\
\hline PIN9 & $\begin{array}{l}35^{\circ} 53^{\prime} 05^{\prime \prime} \mathrm{N} \\
01^{\prime} 39^{\prime} 19^{\prime \prime} \mathrm{E}\end{array}$ & 1320 & SE & $40-50$ & Calcaires & 51 \\
\hline
\end{tabular}

Trois classes d'altitude : altitude < $900 \mathrm{~m}$ (altitude faible); altitude comprise entre $900 \mathrm{~m}$ et $1200 \mathrm{~m}$ (altitude moyenne) ; altitude >1 $200 \mathrm{~m}$ (altitude élevée). Deux types d'exposition : Nord (NNE : Nord-Nord-Est ; NW : Nord-Ouest) et Sud

(SW : Sud-Ouest ; SE : Sud-Est). Trois classes de pente : faible (10-20\%), moyenne (20-40\%), accentuée ( $>40 \%)$.

Trois types de sols : grès, calcaires, schistes. Trois classes d'âge des populations : populations jeunes ( 50 ans); populations moyennement âgées (50-70 ans) ; populations âgées (> 70 ans).

dominants et codominants ont été sondés à cœur à la tarière de Pressler à $1,30 \mathrm{~m}$ au-dessus du sol pour recueillir deux carottes. Les carottes ont été fixées sur des réglettes en bois pour pallier leur fragilité, puis poncées et interdatées (Fritts et Swetnam, 1989). Les épaisseurs des cernes ont été mesurées à l'aide de la table LINTAB (photo 3) couplée au logiciel TSAP $®$ avec une précision de $1 / 100 \mathrm{~mm}$.

\section{Analyse des variations interannuelles des épaisseurs de cernes}

Les épaisseurs des cernes de chaque carotte fournissent une série élémentaire ; la moyenne de deux séries élémentaires représentatives des deux carottes d'un même arbre fournit une série individuelle pour chaque arbre. La chronologie maîtresse de la population résulte de la moyenne, année par année, des épaisseurs des cernes de toutes les séries élémentaires. Les données brutes issues de chaque série chronologique ont fait l'objet des analyses statistiques ci-après (Fritts, 1976 ; Tessier, 1984).

La sensitivité moyenne (SM) mesure la différence entre deux cernes successifs de la même chronologie à leur moyenne ; elle est obtenue par la formule suivante :
$\mathrm{SM}=\sum 2 \times\left(\mathrm{X}_{i}-\mathrm{X}_{\mathrm{i}-1}\right) /\left(\mathrm{X} i+\mathrm{X}_{i-1}\right)$.

avec :

$\mathrm{N}$, nombre de cernes; $\mathrm{X}$, épaisseur du cerne de l'année $n$; $X_{i-1}$, épaisseur du cerne de l'année $n-1$.

Le coefficient d'interdatation (Cl) est le rapport du coefficient moyen de sensitivité d'une chronologie maîtresse à la moyenne des coefficients de sensitivité relatifs aux différentes chronologies individuelles intégrées dans la chronologie maîtresse. Il traduit le bon synchronisme des chronologies moyennes à partir desquelles la chronologie maîtresse a été calculée.

L'autocorrélation (AC) d'ordre $n$ d'une série de cernes est la corrélation qui existe entre celle-ci et la même série décalée de $n$ années dans le passé. Ce coefficient met en évidence l'effet rémanent de facteurs internes et externes à l'arbre au cours de la formation de plusieurs cernes successifs et exprime l'effet de persistance qui caractérise les séries chronologiques (Tessier, 1984).

L'épaisseur moyenne des cernes (EMC) est égale à la somme de toutes les épaisseurs des cernes d'une chronologie divisée par le nombre de cernes. L'écart-type (ET) mesure la 


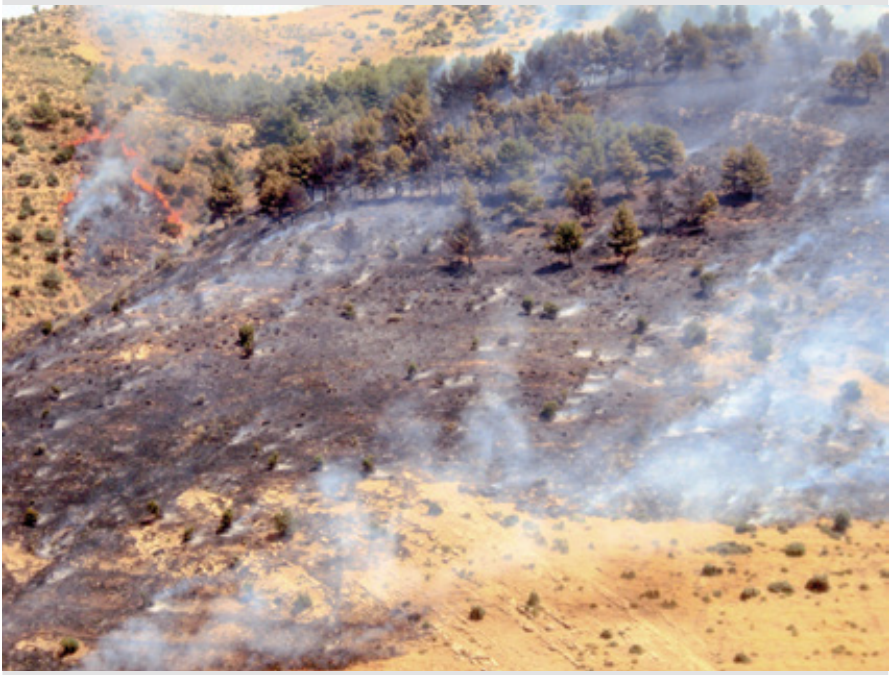

Photo 2.

Les incendies de forêts, une menace récurrente pour les pinèdes de l'Ouarsenis.

Photo M. Sarmoum.

dispersion des valeurs de l'EMC autour de la moyenne. Le coefficient de variation (CV) correspond au rapport de l'écart-type à la moyenne ; ses valeurs sont exprimées en pourcentage (\%).

Pour dégager les variations spatio-temporelles des épaisseurs de cernes, une analyse en composantes principales (ACP) a été d'abord effectuée sur une matrice dans laquelle les individus sont les populations et les variables sont les années, puis sur une matrice dans laquelle les individus sont les populations et les variables sont les différents paramètres statistiques calculés.

\section{Standardisation des épaisseurs des cernes}

La standardisation a pour objectif d'éliminer des signaux autres que le signal climatique tel l'effet de l'âge (Cook et al., 1990). Les épaisseurs brutes ont été standardisées par la méthode des filtres (fenêtre de 15 ans) à l'aide du package PPPBase (Guiot et Goeury, 1996). Pour chaque valeur $X_{t}$ de la croissance réelle est attribuée une valeur théorique $Y_{t}$ extraite de la courbe d'ajustement. Un indice de croissance annuelle $\left(\mathrm{l}_{\mathrm{a}}\right)$ est calculé par la formule (Fritts, 1976) $: l_{a}=X_{t} / Y_{t}$.

Après indexation, nous avons procédé à la recherche des cernes caractéristiques de la chronologie maîtresse. De tels cernes témoignent des conditions particulières qui ont agi positivement (cerne épais) ou négativement (cerne mince) sur la croissance des arbres (Fritts, 1976). Pour les définir, la plupart des auteurs utilisent des méthodes statistiques qui tiennent compte de la moyenne et de son écart-type (Safar, 1994 ; El Khorchani, 2006). Si les valeurs de l'indice se distribuent autour d'une valeur proche de la moyenne $\left(l_{m}\right) \pm$ écart-type $(E T)$, les valeurs sont considérées comme ordinaires. Le recours à la valeur $\pm 0,5$ écart-type est lié à la forte variabilité interannuelle des épaisseurs de cernes dans nos séries chronologiques. Dans ce cadre, l'identification d'un cerne mince ou épais se fait selon la formule suivante (El Khorchani et al., 2007) :

- le cerne est mince si $l_{a}<l_{m}-0,5 E T$;

- il est épais si $l_{a}>l_{m}+0,5 E T$.

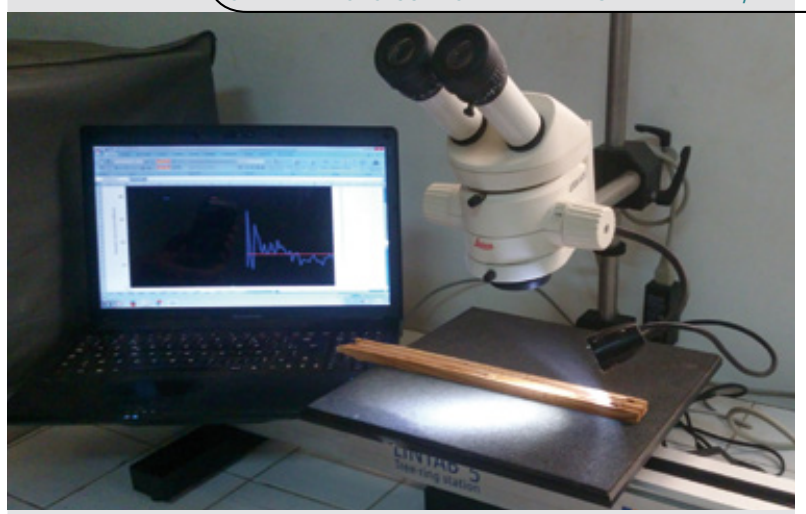

Photo 3.

Dispositif d'interdatation et de mesure des épaisseurs de cernes.

Photo M. Sarmoum.

\section{Relation cerne-climat}

Les relations cerne-climat ont été établies par le calcul des fonctions de réponse sur la période 1967-2010 à l'aide du package PPPBase (Guiot et Goeury, 1996). Dans cette régression orthogonalisée, les variables dépendantes sont les résidus obtenus par l'application de la méthode ARMA ( $\mathrm{Au}$ toRegressives Moving Average). Les régresseurs climatiques utilisés sont les précipitations mensuelles ( $\mathrm{Pm})$, les températures moyennes maximales " $M$ » et les températures moyennes minimales « $\mathrm{m}$ » sur une période de 12 mois allant du mois d'octobre de l'année $n-1$ au mois de septembre de l'année $n$ (Serre-Bachet, 1982). Les données climatiques utilisées couvrent la période 1967-2010, période commune entre toutes les populations. Ces données concernent les stations de Theniet El Had (PIN1, PIN2 et PIN3), Chlef (PIN4 et PIN5) et Tiaret (PIN6, PIN7, PIN8 et PIN9).

La fiabilité de la relation entre la croissance radiale et les régresseurs climatiques (variables climatiques mensuelles) est fournie par le rapport de chaque coefficient de régression partiel à son écart-type $(\mathrm{R} / \mathrm{s})$. Si l'on suppose que ces rapports $(R / s)$ suivent une distribution normale, leur niveau de signification est établi selon les seuils de cette loi (tableau II). Afin de faciliter la comparaison entre les fonctions de réponse, les seuils de signification des coefficients de régression sont codés de 0 à 4 . Seules les fonctions de réponse significatives ( $p$ $<0,05)$ ont été prises en considération pour l'interprétation des relations cerne-climat (Messaoudene, 1989).

Tableau II.

Signification globale des fonctions de réponse en fonction du rapport $\mathrm{R} / \mathrm{s}$.

\begin{tabular}{|c|c|c|}
\hline$R / S$ & Code & Signification \\
\hline $1,65-1,96$ & 1 & $90 \%$ \\
\hline $1,96-2,58$ & 2 & $95 \%$ \\
\hline $2,58-3,29$ & 3 & $99 \%$ \\
\hline 3,29 & 4 & $99,9 \%$ \\
\hline
\end{tabular}




\section{Résultats et discussion}

\section{Variations spatio-temporelles des épaisseurs des cernes}

Les courbes maîtresses représentatives des chronologies moyennes par population mettent en évidence une forte variabilité interannuelle de la croissance radiale et une tendance générale à la diminution de celle-ci avec l'âge (figure 2). Cette tendance définit les variations de basse fréquence enregistrées sur toute la chronologie. Les variations de moyenne fréquence (à l'échelle d'une décennie) se traduisent tantôt par des phases favorables à la croissance (1970-1979), tantôt par un déclin de la croissance radiale (1942-1947, 1984-1994, 2000-2004). Ces phases sont synchrones pour la majorité des populations et suggèrent l'influence d'un facteur commun sur la croissance radiale qui peut être d'origine climatique. Les variations de haute fréquence (échelle annuelle) sont exprimées par des cernes très larges ou au contraire très minces, et traduisent la variabilité interannuelle des conditions qui agissent sur la croissance radiale (climat, attaque de parasites, coupes illicites...).

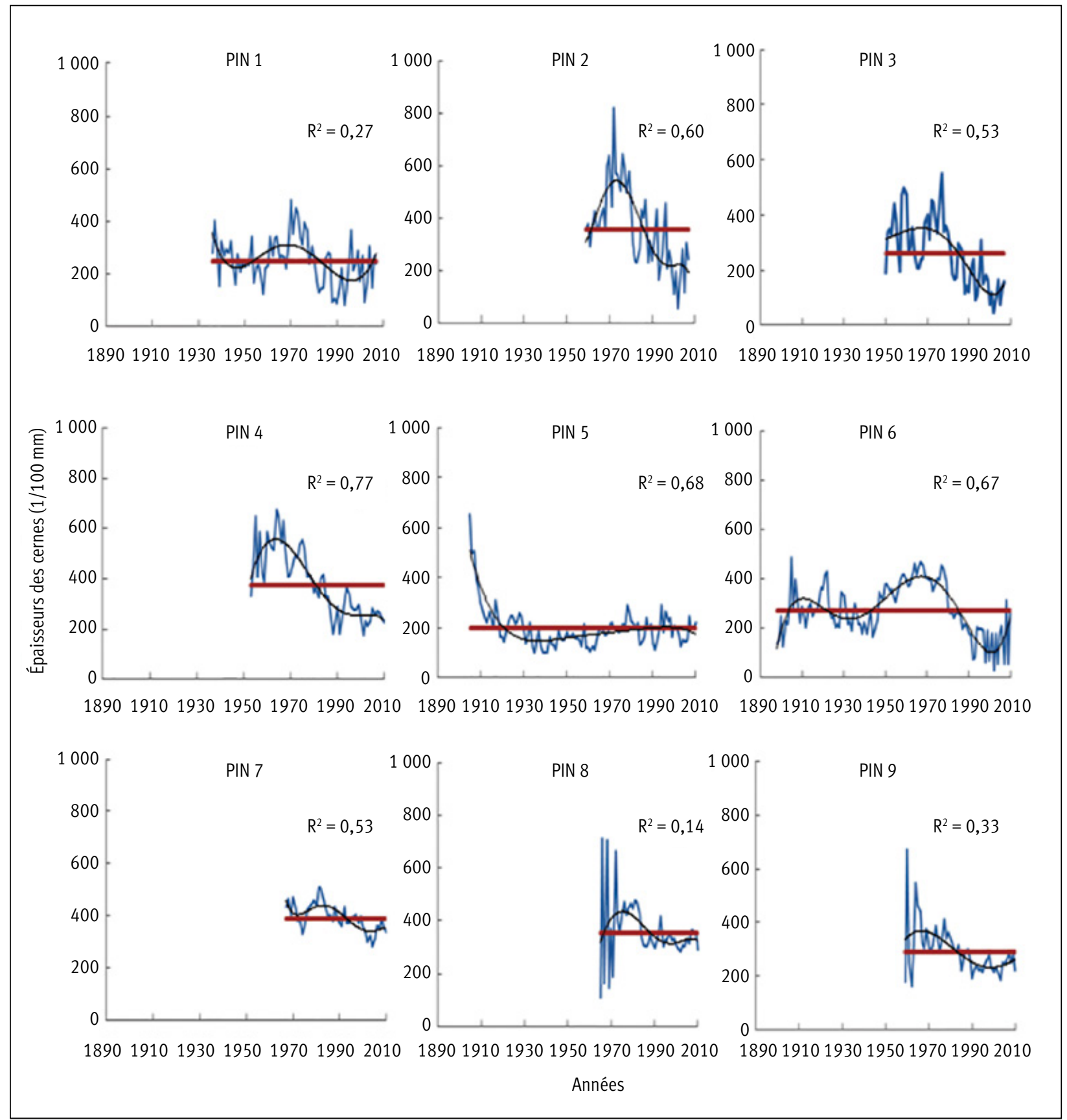

Figure 2.

Variations spatio-temporelles des épaisseurs brutes des cernes des chronologies du pin d'Alep. Les variations interannuelles des épaisseurs de cernes sont représentées par le trait bleu. Le trait rouge représente la moyenne des épaisseurs de cernes. Le trait noir représente la fonction d'ajustement associée à son coefficient de détermination $\left(R^{2}\right)$. 


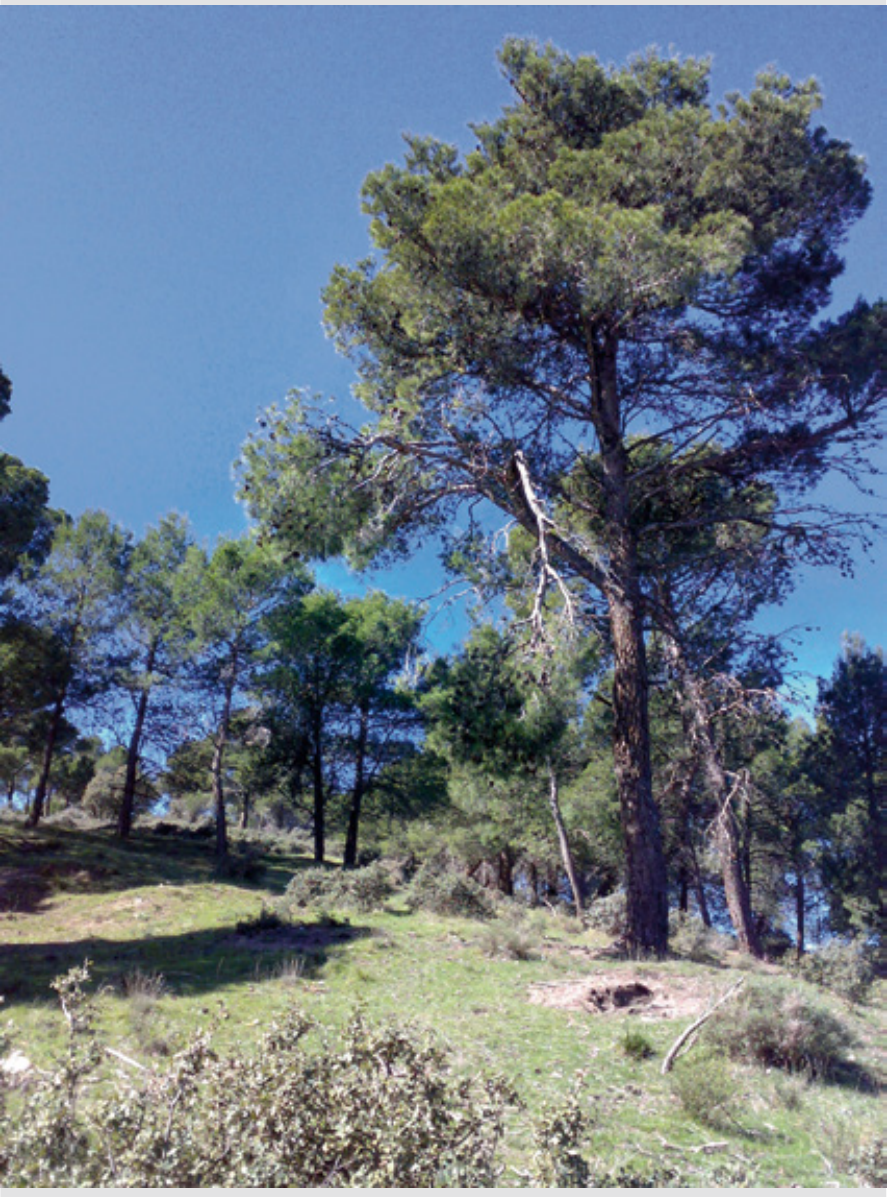

Photo 4.

Pinède dégradée suite aux coupes illicites et aux incendies, Ouarsenis.

Photo M. Sarmoum.
Certaines populations manifestent des patrons de croissance particuliers, telle la population PIN5 pour laquelle un déclin de la croissance a été enregistré entre les années 1930 et 1960, et la population PIN6 qui manifeste une augmentation de la croissance radiale entre les années 1950 et 1970. Ces variations qui n'affectent qu'une seule population peuvent être difficilement attribuées aux variables climatiques, mais plutôt à l'action anthropique (coupes illicites, gemmage) (Boudy, 1950 ; BNEDER, 2009) (photo 4).

Les paramètres statistiques calculés sur les chronologies maîtresses (tableau III), représentatives de chaque population, montrent de fortes différences d'une population à l'autre. Les valeurs de l'EMC varient de $1,98 \mathrm{~mm} / \mathrm{an}$ (PIN5) à $3,95 \mathrm{~mm} / \mathrm{an}$ (PIN7). Les valeurs de l'écart-type (ET) et du coefficient de variation (CV) indiquent une forte variabilité interannuelle de la croissance radiale due aux fluctuations des facteurs aléatoires qui agissent sur la croissance radiale (climat, coupes illicites, attaques de parasites), mais aussi l'effet de l'âge. En effet, les populations âgées (PIN5 et PIN6) présentent des épaisseurs moyennes inférieures à celles des populations jeunes (PIN7 et PIN8) (figure 3a). La comparaison de l'EMC avec les autres pinèdes méditerranéennes montre des valeurs nettement supérieures à celles obtenues dans l'Atlas saharien (Safar, 1994), assez élevées par rapport à celles du Sud-Est de la France (Serre-Bachet, 1982), et conformes à celles obtenues en Grèce (Papadopoulos et al., 2001) et en Kroumirie (El Khorchani, 2006).

Le coefficient d'autocorrélation varie de 0,187 (PIN6) à 0,286 (PIN2). Dans l'ensemble, ces valeurs reflètent un effet de persistance faible et indiquent que l'épaisseur du cerne dépend en grande partie des conditions climatiques de l'année en cours ; l'effet des années précédentes est secondaire. Toutefois, nous pouvons constater un effet négatif de l'âge sur le phénomène de persistance car les populations âgées (PIN5 et PIN6) affichent les valeurs les plus faibles (figure 3b).

Les valeurs de la sensitivité moyenne des chronologies maîtresses varient de 0,17 (PIN7) à 0,33 (PIN2). Les populations PIN4, PIN5, PIN7 sont peu sensitives, comme l'attestent les valeurs inférieures à 0,20 (Fritts, 1976). Les autres populations sont plutôt sensitives (avec $S M>0,20$ ). Les populations les plus sensitives se localisent sur des substrats gréseux (PIN1, PIN2 et PIN3) ou schisteux (PIN6), tandis que les populations les moins sensitives sont installées sur substrat calcaire (PIN4, PIN7). Ces résultats confirment ceux obtenus par Papadopoulos et al. (2001) pour les peuplements de pin d'Alep en Grèce. Par ailleurs, les valeurs de sensitivité moyenne enregistrées dans la zone d'étude corroborent celles obtenues pour cette espèce dans l'ensemble du bassin méditerranéen (Serre-Bachet, 1982 ; Safar, 1994 ; Nicault, 1999 ; Papadopoulos et al., 2001 ; El Khorchani, 2006).

EMC : épaisseur moyenne des cernes (mm/an) ; ET : écart-type

$\mathrm{CV}$ : coefficient de variation (\%) ; SM : sensibilité moyenne ;

$\mathrm{Cl}$ : coefficient d'interdatation ; AC : coefficient d'autocorrélation. 


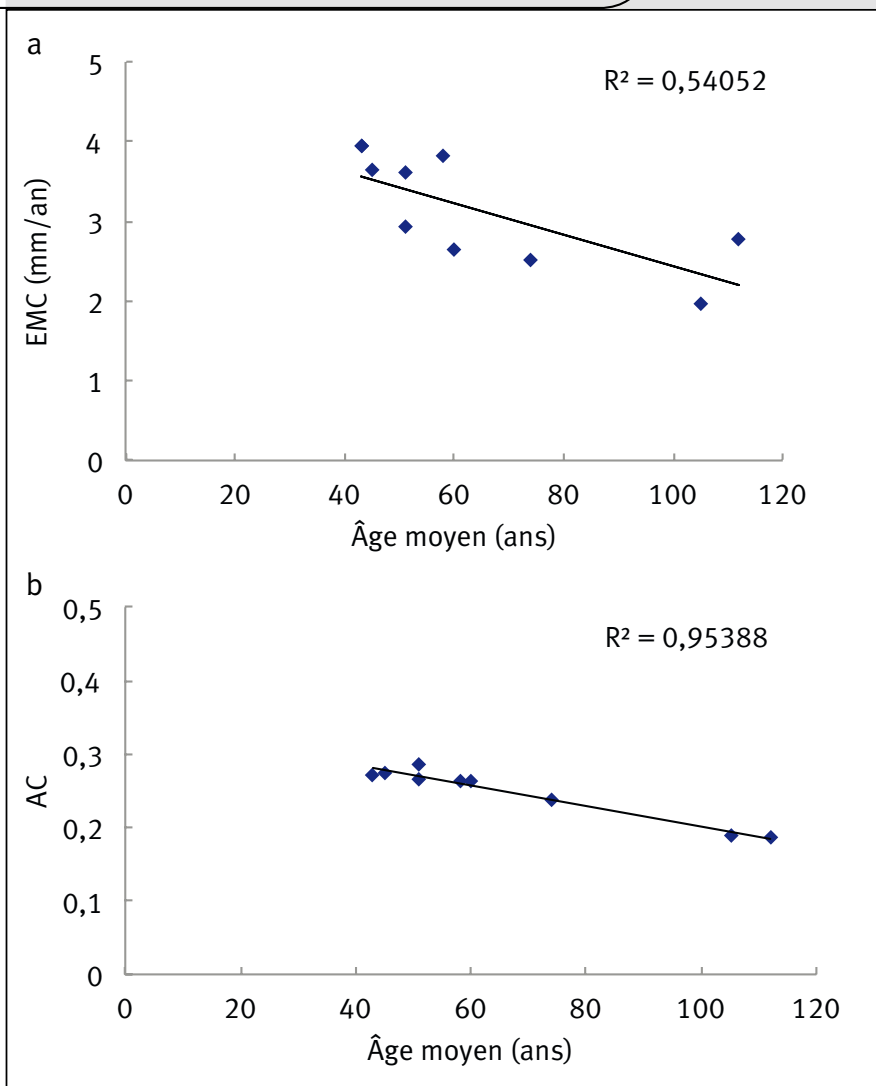

Figure 3.

Effet négatif de l'âge des arbres sur l'épaisseur moyenne de cerne (EMC) (graphe a) et sur les autocorrélations (AC) (graphe b) des populations étudiées.

Les valeurs du coefficient d'interdatation varient de 0,62 à 0,85 . La comparaison des valeurs met en évidence un bon synchronisme des séries individuelles lors de l'interdatation, ce qui traduit un comportement homogène des arbres vis-à-vis des variations climatiques interannuelles (Messaoudene, 1989). Ce constat concerne en particulier les populations PIN1, PIN2 et PIN3 (substrat gréseux) dont le coefficient d'interdatation est supérieur à 0,80 et la population PIN6 installée sur schistes $(0,73)$. Les valeurs assez faibles $(<0,70)$ pour les populations installées sur calcaire (PIN4, PIN5, PIN7, PIN8 et PIN9) dénotent une hétérogénéité marquée d'un arbre à l'autre vis-à-vis de la réponse au climat (Tessier, 1984).

La projection des populations en fonction des variations interannuelles des épaisseurs de cernes montre une faible discrimination entre celles-ci (figure 4a). Cette discrimination se fait par l'axe 2 qui explique $14 \%$ de la variabilité totale et met en évidence l'existence de deux groupes de populations, PIN1, PIN2 et PIN3 situées sur le côté positif de l'axe 2 et PIN5, PIN7 et PIN8 situées sur le côté négatif. Les populations PIN1, PIN2 et PIN3 très proches sur le plan géographique et installées sur le même substrat (grès) traduisent un effet homogène du climat sur la variabilité interannuelle de la croissance, comme l'attestent des sensitivités moyennes et des coefficients d'interdatation élevés. À l'inverse, les autres populations, installées sur substrat calcaire, sont affectées par des sensitivités moyennes et des coefficients d'interdatation plus faibles.
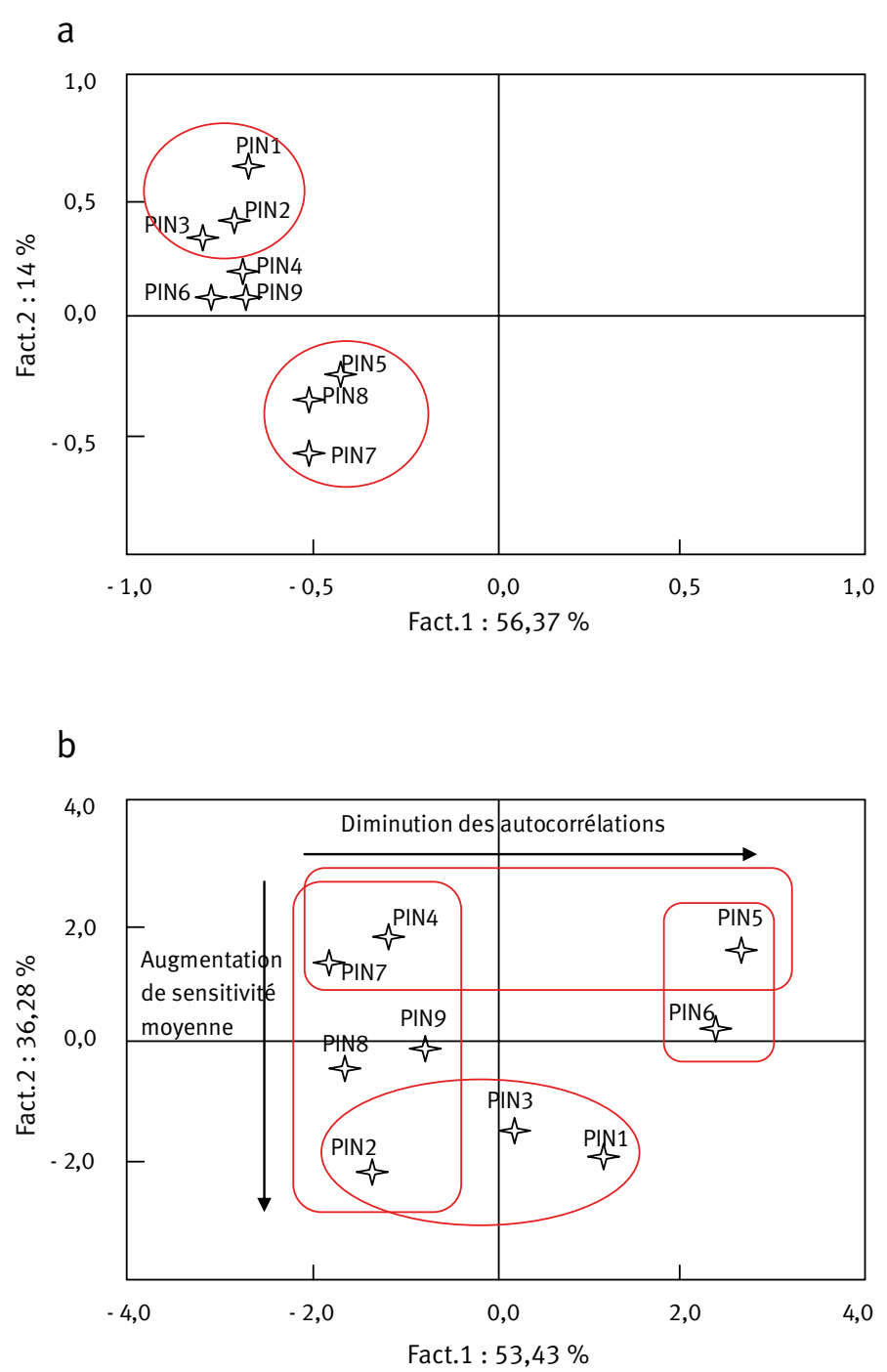

Figure 4.

Projection des populations dans l'ACP (axes 1 et 2), en fonction des épaisseurs de cernes (graphe a) et en fonction des paramètres statistiques calculés (graphe b).

Lorsque les variables prises en compte sont les valeurs des différents paramètres statistiques, la projection des populations sur les plans matérialisés par les axes 1 et 2 (figure 4b) met en évidence quatre groupes :

- PIN1, PIN2 et PIN3 : populations installées sur substrat gréseux, à sensitivité moyenne et coefficient d'interdatation élevé ;

- PIN4 et PIN7 : populations installées sur substrat calcaire, à sensitivité moyenne et coefficient d'interdatation faible ;

- PIN5 et PIN6 : populations âgées à faible coefficient d'autocorrélation ;

- PIN2, PIN4, PIN7, PIN8 et PIN9 : populations jeunes à fort coefficient d'autocorrélation. 


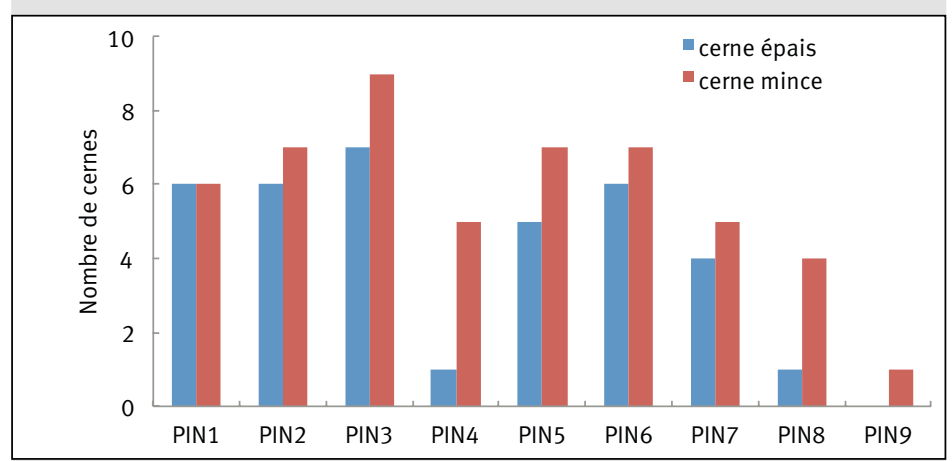

Figure 5.

Nombre de cernes minces et de cernes épais chez les populations du pin d'Alep sur la période 1967-2010.

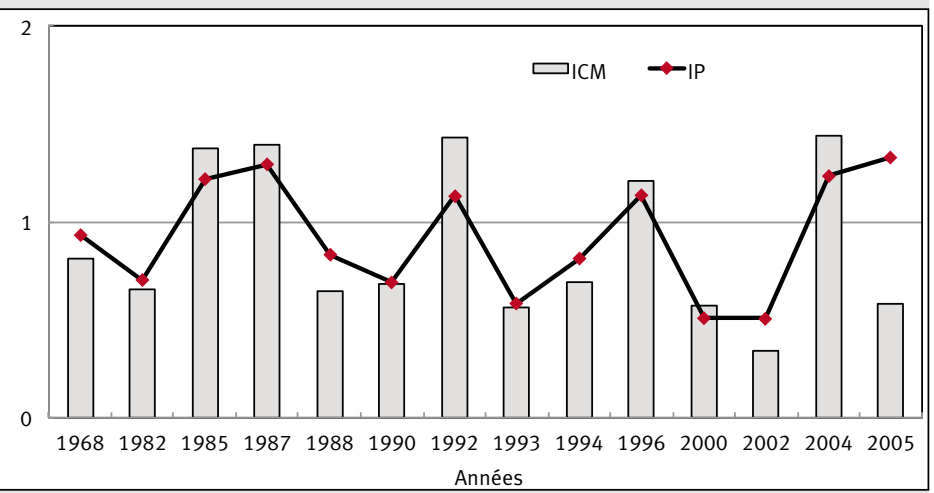

Figure 6.

Relation entre l'indice de précipitations (IP) et l'indice moyen de croissance (ICM) des populations du pin d'Alep.

IP est le rapport entre les précipitations annuelles de l'année $n$ et la moyenne de la période 1967-2010.

ICM est l'indice moyen de croissance de l'année $n$ de toutes les chronologies standardisées.

\section{Identification des cernes caractéristiques}

Les populations enregistrent au total 22 cernes caractéristiques au cours de la période commune 1967-2010, dont 14 sont communs pour au moins trois populations. Les cernes épais correspondent à 1985, 1987, 1992, 1996 et 2004. Les cernes minces relèvent de 1968, 1982, 1988, 1990, 1993, 1994, 2000, 2002 et 2005. Chez la plupart des populations, le nombre de cernes minces est supérieur au nombre de cernes épais (figure 5) ; ce résultat traduit la tendance à la diminution de la croissance radiale ces dernières décennies, qui devient plus marquée à partir des années 1980. Il est à noter aussi que le nombre de cernes caractéristiques varie d'une population à une autre. Les populations âgées (PIN5 et PIN6) ou celles localisées sur un substrat gréseux (PIN1, PIN2 et PIN3) recouvrent un nombre élevé de cernes caractéristiques. En revanche, les populations jeunes et/ou celles localisées sur un substrat calcaire (PIN4, PIN7, PIN8 et PIN9) présentent un nombre plus faible de cernes caractéristiques.

La confrontation entre les cernes caractéristiques (épais ou minces) et les données météorologiques met en évidence deux types de relation (figure 6) :

- relation de type " année humide - cerne épais »

(années 1985, 1987, 1996 et 2004);

- relation de type « année sèche - cerne mince »

(années 1968, 1982, 1988, 1990, 1993, 2000 et 2002).

\section{Relations cerne-climat}

Les résultats des fonctions de réponse (figure 7) montrent que l'épaisseur des cernes du pin d'Alep est positivement reliée aux précipitations de la période précédant ou contemporaine de la formation du cerne (octobre de l'année $n$ - 1 à mai de l'année $n$ ). Des relations négatives apparaissent avec les précipitations de janvier (PIN7), juin (PIN5), août et septembre (PIN4). Les relations positives s'expliquent par un besoin élevé en eau lors de la formation du cerne et par la reconstitution des réserves hydriques du sol avant sa formation (Papadopoulos et al., 2001). Les relations négatives avec les précipitations de janvier peuvent être expliquées par un engorgement du sol par l'eau ou une accumulation de la neige qui retarde l'activité cambiale (Messaoudene, 1989), celles de juin, août et septembre par l'augmentation de l'évapotranspiration (Safar, 1994). Les relations négatives avec les précipitations concernent les populations installées sur calcaire (PIN4, PIN5 et PIN7). Le nombre de mois au cours desquels les relations précipitations-croissance sont significatives est plus élevé chez les populations installées sur substrat gréseux ou dans le versant sud.

Les températures maximales sont le plus souvent négativement corrélées à la croissance radiale durant la période janvier-septembre. Des températures élevées au cours de ces mois augmentent l'évapotranspiration et exposent la plante à un stress hydrique (Serre-Bachet, 1982). Les relations positives mettent en jeu les mois de novembre-décembre (PIN2, PIN6, PIN7 et PIN8) et janvier-février (PIN9) et pourraient s'expliquer par le fait que des températures élevées en automne et en hiver favorisent la rhizogenèse et améliorent la capacité d'absorption de l'eau par la plante (Meddour, 1992).

Les températures minimales sont positivement corrélées à l'épaisseur des cernes au cours des mois de janvier et février pour la plupart des populations, avril-mai (PIN4, PIN7) et juin (PIN4, PIN8). Des relations négatives avec les températures minimales sont mises en évidence en toutes saisons : octobre-novembre (PIN8, PIN9), décembre-janvier (PIN4), mars-avril (PIN1, PIN2, PIN3), mai-juin (PIN3, PIN7, PIN9) et août-septembre (PIN4). Ces résultats confirment que le pin d'Alep redoute les froids hivernaux et les froids tardifs du début de printemps qui retardent le démarrage de l'activité cambiale (Serre-Bachet, 1982 ; Nicault et al., 2001).

L'étude des fonctions de réponse du pin d'Alep dans l'ensemble du pourtour méditerranéen (de Luis et al., 2013) a révélé que les températures hivernales agissent positivement sur la croissance radiale du pin d'Alep vers la limite nord de son aire géographique et négativement vers la limite sud. Dans ce contexte, le réchauffement climatique actuel et futur (GIEC, 2007) pourrait jouer un rôle positif sur la croissance du pin d'Alep dans les sites humides et négatif dans les sites secs (El Khorchani et al., 2007). 


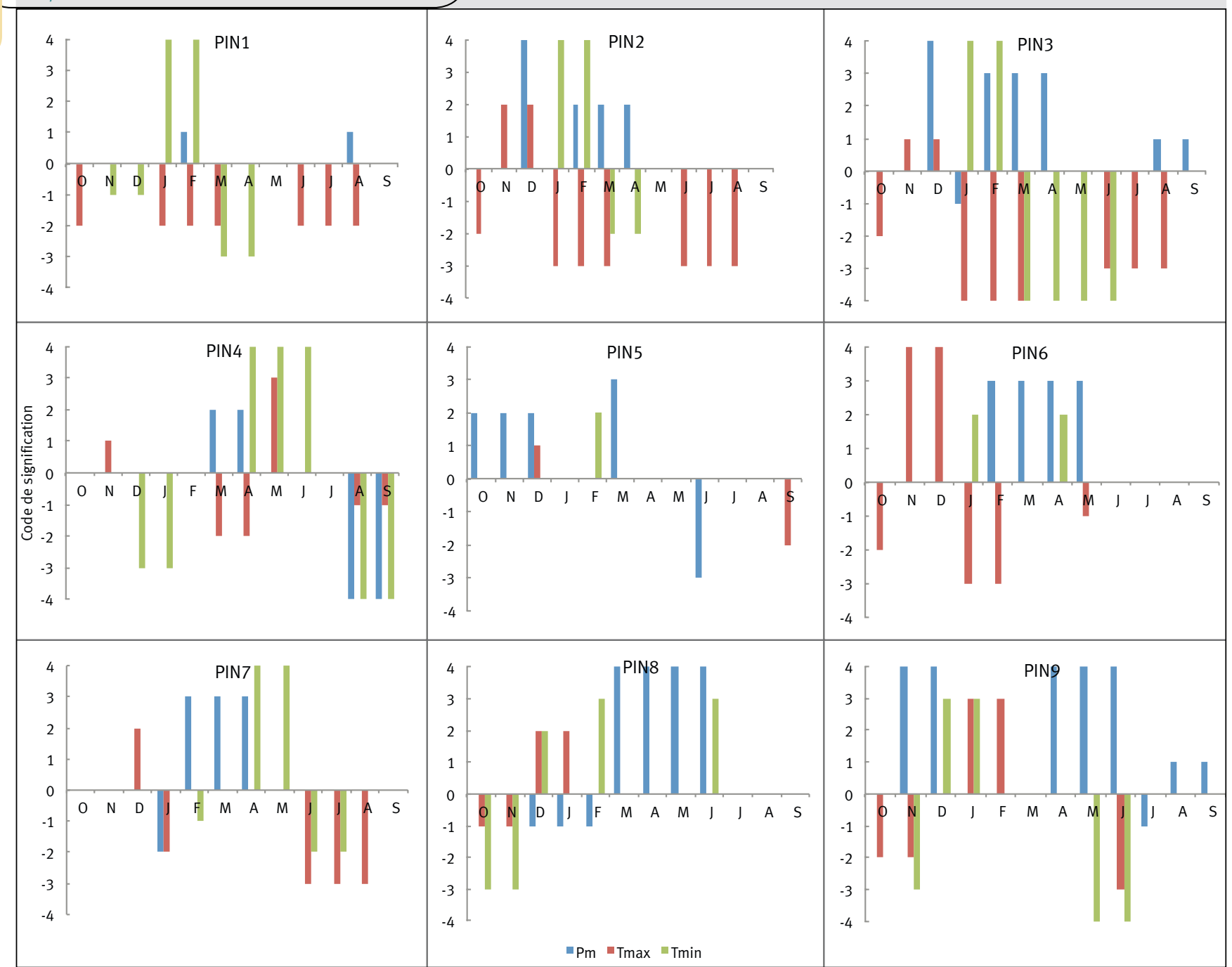

Figure 7.

Relations cerne-climat chez les populations du pin d'Alep dans la zone d'étude. Les codes de signification représentent le rapport entre le coefficient de régression $(\mathrm{R})$ et son écart-type (s) pour chaque variable climatique ( $\mathrm{Pm}$ : précipitations mensuelles ; Tmax : température mensuelle maximale ; Tmin : température mensuelle minimale). Les signes de signification $(-$ ou +$)$ indiquent respectivement des relations négatives ou positives avec la croissance radiale. Les lettres en abscisses représentent les mois de l'année allant d'octobre de l'année $n-1$ à septembre de l'année $n$.

\section{Conclusion}

Le présent travail constitue une première approche de l'étude des relations entre le climat et la croissance radiale du pin d'Alep en fonction des facteurs stationnels dans le massif de l'Ouarsenis.

Les patrons qui régissent la croissance radiale du pin d'Alep dans la zone d'étude sont le climat, la nature du substrat et l'exposition. L'âge des arbres joue un rôle négatif sur la croissance radiale et sur l'effet de persistance. L'étude met aussi en évidence une sensibilité aux précipitations durant plusieurs mois de l'année, modulée selon les facteurs stationnels et plus marquée chez les populations installées en versant sud et/ou sur substrat gréseux que chez celles installées en versant nord et/ou sur substrat calcaire. Les températures agissent souvent négativement sur la croissance radiale aussi bien par les valeurs maximales que minimales. Ces résultats montrent que les facteurs stationnels influencent significativement la réponse du pin d’Alep aux changements climatiques et déterminent par conséquent sa plasticité écologique.
Dans le contexte des changements climatiques futurs allant vers une augmentation des températures et une diminution des précipitations, les résultats de cette étude dressent un diagnostic qui demande à être étendu à d'autres populations de pin d'Alep installées dans des conditions de biotopes différentes pour comprendre sa réponse aux changements climatiques et améliorer les connaissances en matière de conduite des peuplements et les futurs projets de reboisements.

\section{Remerciements}

Ce travail a bénéficié du soutien financier du ministère algérien de l'Enseignement supérieur et de la Recherche scientifique (PNR-MESRS) et de l'Université Ibn Khaldoun (Tiaret) pour l'attribution d'un financement de stage de courte durée. Nous tenons à remercier la direction du Parc national de Theniet El Had et les conservations des forêts de l'Ouarsenis pour avoir facilité le travail de terrain, ainsi que trois relecteurs anonymes pour leurs critiques et remarques très constructives. 


\section{Références bibliographiques}

Benabadji N., Bouazza M., 2000. Contribution à une étude bioclimatique de la steppe à Artemisia herba-alba Asso dans l'Oranie (Algérie occidentale). Sécheresse, 11 (2) : 117-123.

Bensaid S., 1995. Bilan critique du barrage vert en Algérie. Sécheresse, 6 (3) : 247-255.

BNEDER (Bureau national d'études pour le développement rural), 2009. Plan national de développement forestier (PNDF). Rapport de synthèse nationale. Alger, Algérie, BNEDER, 87 p.

Boudy P., 1950. Économie forestière nord-africaine. Tome 2 : Monographies et traitements des essences forestières. Paris, France, Larose, 523 p.

Charru M., Seynave I., Morneau F., Bontemps J.-D., 2010. Recent changes in forest productivity: An analysis of national forest inventory data for common beech (Fagus sylvatica L.) in north-eastern France. Forest Ecology and Management, 260: 864-874.

Cook E., Briffa K., Shiyatov S., Mazepa V., 1990. Tree rings standardization and growth-trend estimation. In: Cook E., Kairiukstis V. (eds). Methods of dendrochronology. Applications in the environnemental sciences. Dordrecht, Pays-Bas, Kluwer Academic Publ., 104-123.

Coomes D. A., Burslem D. F., Simonson W. D., 2014. Forests and Global Change. Cambriidge, Royaume-Uni, Cambridge University Press, $473 \mathrm{p}$.

de Luis M., Cufar K., Di Filippo A., Novak K., Papadopoulos A. et al., 2013. Plasticity in dendroclimatic response across the distribution range of Aleppo pine (Pinus halepensis). Plos One, 8 (12): e83550. doi:10.1371/journal.pone.0083550.

El Khorchani A., 2006. Approche dendrochronologique de l'influence des changements climatiques sur la productivité des forêts de pin d'Alep en Tunisie. Thèse de doctorat, Université d'Aix-Marseille III, France, 217 p.

El Khorchani A., Gadbin-Henry C., Khaldi A., 2007. Impact de la sécheresse sur la croissance de trois espèces forestières en Tunisie (Pinus halepensis Mill., Pinus pinea L. et Pinus pinaster Sol.). Sécheresse, 18 (2) : 113-121.

Fritts H. C., 1976. Tree rings and climate. Londres, Royaume-Uni, Academic Press, $567 \mathrm{p}$.

Fritts H. C., Swetnam T. W., 1989. Dendroecology: A tool for evaluating variations in past and present forest environments. Advances in Ecological Research, 19: 111-188.

GIEC, 2007. Bilan 2007 des changements climatiques : les bases scientifiques physiques. Rapport du Groupe de travail I du GIEC. Genève, Suisse, $27 \mathrm{p}$.

Giorgi F., Lionello P., 2008. Climate change projections for the Mediterranean region. Global and Planetary Change, 63: 90-104.

Guiot J., Goeury C., 1996. PPPBase, a software for statistical analysis of paleoecological and paleoclimatological data. Dendrochronologia, 14: 295-300.

Hirche A., Boughani A., Salamani M., 2007. Évolution de la pluviosité annuelle dans quelques stations arides algériennes. Sécheresse, 18 (4) : 314-320.

Jonard M., Colmant R., Heylen C., Ysebaert C., Carton C., Picard L. et al., 2014. Impact de boisements résineux sur la séquestration du carbone dans les Andes péruviennes : cas des plantations de Pinus patula dans la coopérative de Granja Porcon (Cajamarca), Pérou. Bois et Forêts des Tropiques, 322 (4) : 17-27. http://bft.cirad.fr/cd/ BFT 322 17-27.pdf
Kadik B., 1983. Contribution à l'étude du Pin d'Alep (Pinus halepensis Mill.) en Algérie : écologie, dendrométrie, morphologie. Doctorat d’État, Université Paul Cézanne, France, 623 p.

Mattauer M., 1958. Étude géologique de l'Ouarsenis oriental (Algérie). Alger, Algérie, Service de la Carte géologique de l’Algérie, $550 \mathrm{p}$.

Meddi M., Toumi S., 2013. Study of the interannual rainfall variability in northern Algeria. Revue Scientifique et Technique, LJEE, 23 : 40-59.

Meddour H., 1992. Contribution à l'étude dendrochronologique de deux provenances de pin d'Alep (Pinus halepensis) en Algérie. Cas d'une station littorale et d'une autre continentale. Thèse de magistère. INA, Algérie, 178 p.

Messaoudene M., 1989. Dendroécologie et productivité de Quercus afares Pomel et Quercus canariensis Willd. dans les massifs forestiers de l'Akfadou et de Beni Ghorbi en Algérie. Thèse de doctorat, Université d'Aix-Marseille III, France, 128 p.

Messaoudene M., Rabhi K., Megdoud A., Sarmoum M., Dahmani-Megrerouche M., 2013. État des lieux et perspectives des cédraies algériennes. Forêt Méditerranéenne, 34 (4) : 1-8.

Nicault A., 1999. Analyse de l'influence du climat sur les variations inter et intra-annuelles de la croissance radiale du pin d'Alep (Pinus halepensis Mill.) en Provence calcaire. Thèse de doctorat, Université d'Aix-Marseille III, France, 256 p.

Nicault A., Rathgber C., Tessier L., Thomas A., 2001. Observation sur la mise en place du cerne chez le pin d'Alep (Pinus halepensis Mill.) : confrontation entre les mesures de croissance radiale, de densité et les facteurs climatiques. Annales des Sciences Forestières, 58 : 769-784.

Papadopoulos A., Serre-Bachet F., Tessier L., 2001. Tree ring to climate relationships of Aleppo pine (Pinus halepensis Mill.) in Greece. Ecologia Mediterranea, 27 (1): 89-98.

Quézel P., 2000. Réflexions sur l'évolution de la flore et de la végétation au Maghreb méditerranéen. Paris, France, Ibis Press, 117 p.

Rabhi K., Tafer M., Messaoudene M., 2014. Évolution des proportions d'aubier et de duramen du cèdre de l'Atlas, Cedrus atlantica Manetti, en Algérie. Bois et Forêts des Tropiques, 322 (4) : 77-87. http://bft.cirad.fr/cd/BF_ 322_77-87.pdf

Safar W., 1994. Contribution à l'étude dendroécologique du pin d'Alep (Pinus halepensis Mill.) dans une région semi-aride d'Algérie : l'Atlas Saharien (Ouled Naïl, Aurès, Hodna). Thèse de doctorat, Université d'Aix-Marseille III, France, 225 p.

Sari Dj., 1977. L'Homme et l'érosion dans l'Ouarsenis. Alger, Algérie, SNED, 546 p.

Sarmoum M., 2008. Impact du climat sur le dépérissement du cèdre de l'Atlas. Diagnostic climatique et dendroécologique de la cédraie de Theniet El Had. Thèse de magister, USTHB, Algérie, 98 p.

Saxe H., Cannell M., Johnsen O., Ryan M. G., Vourlitis G., 2001. Tree and forest functioning in response to global warming. New Phytologist, 149: 369-400.

Serre-Bachet F., 1982. Analyse dendroclimatologique comparée de quatre espèces de pin et du chêne pubescent dans la région de la Gardiole près de Rians (Var, France). Ecologia Mediterranea, 8 : 167-183.

Tessier L., 1984. Dendroclimatologie et écologie de Pinus silvestris L. et Quercus pubescens Willd. dans le sud-est de la France. Thèse de doctorat, Université d'Aix-Marseille III, France, 275 p.

Thuiller W., Albert C., Araújo M. B., Berry P. M., Cabeza M., Guisan A. et al., 2008. Predicting global change impacts on plant species' distributions: future challenges. Perspectives in Plant Ecology, Evolution and Systematics, 9: 137-152. 